\title{
EFFECTIVENESS OF EDUCATIONAL INTERVENTION ON REHABILITATION KNOWLEDGE AND PRACTICE AMONG PATIENTS AFTER HIP REPLACEMENT SURGERY
}

\author{
${ }^{1}$ Hoang Van Tuan, ${ }^{2}$ Truong Tuan Anh \\ ${ }^{1}$ Thanh Hoa Medical College; ${ }^{2}$ Nam Dinh University of Nursing
}

\begin{abstract}
Objective: To examine the effectiveness of educational intervention on rehabilitation knowledge and practice among patients after hip replacement surgery at Thanh Hoa General Hospital. Method: An educational intervention study with comparison of rehabilitation knowledge and practice at baseline and post intervention among 55 patients after hip replacement surgery. Results: The mean score of rehabilitation knowledge at immediatly after the intervention increased to $13.18 \pm 2.74$ compared to $7.96 \pm 1.97$ the baseline $(p<0.001)$. The proportion of study participants who achieved rehabilitation knowledge increased to $87,3 \%$ after the intervention. The mean score of practice at immediatly after the intervention increased significantly, reaching 13.60 2.37 compared to $6.22 \pm 2.37$ at the baseline $(p<0.001)$. The proportion of study participants who achieved the rehabilitation practice increased to $90,9 \%$ after the intervention. Conclusion: The rehabilitation knowledge and practice among patients after hip replacement surgery were still limited before intervention. Educational interventions significantly improved rehabilitation knowledge and practice among patients after hip replacement surgery. The nurses and healthcare providers should consider to integrate the educational intervention in their usual care practice for the patients with hip replacement surgery.
\end{abstract}

Keywords: Hip replacement, rehabilitation, knowledge, practice, Thanh Hoa.

\section{INTRODUCTION}

Currently, along with the development of medicine, the aging of the global population is increasing. Diseases in the hip joint are more common, in which femoral neck fractures and hip osteoarthritis are the most common diseases. According to statistics of the World Health Organization, one in eight people had a disease in the hip joints and hip replacement surgery was the optimal indication when all treatments fail. Worldwide, more than 1 million hip replacements were performed each year. In 2015, the number of hip replacement surgeries per 100,000 population was 308 in Sweden, 299 in Germany, 241 in France, and 204 in the US [1]. In Vietnam, there are currently no specific statistics on the number of hip replacements, but research showed that hip replacement surgery become popular in Vietnam and become a routine surgery in hospitals.

The financial burden of hip replacement in health care was high, with in the United
Cor. author: Hoang Van Tuan

Address: Thanh Hoa Medical College

Email: tuanngoaicdy@gmail.com
Received: Jan 09, 2022

Accepted: Feb 26, 2022

Published: Feb 27, 2022 
States alone it was estimated that more than $\$ 15$ billion a year was spent on hip replacement surgery [2]. In Vietnam, the cost for a hip replacement surgery ranged from 45-80 VND million excluding hospital fees and other costs, although the cost come from a flexible health insurance policy, the financial burden for people with health insurance was reduced. This payment level was still relatively high compared to the income of patients who mainly live in rural areas, accounting for $67 \%$ [3].

The fact that taking care of hip replacement patients before and after surgery indicated that there were challenges. Before surgery due to pain, legs are sedentary for many months and years, causing muscle atrophy in buttocks, thighs, making muscles weaker than usual. After surgery, the pain of the incision was the most affected in the first three days [4], in addition, we also found that the patient's movement depended mostly on the patient's own efforts, the patients were afraid of pain, fatigue, therefore, they were scare of rehabilitation, along with the lack of knowledge about rehabilitation after surgery, affecting the recovery process after surgery. Stemming from the fact of care, the question is whether hip replacement patients have a full awareness of rehabilitation and the need for action to improve for the purpose of improving rehabilitation knowledge and practice for patients with hip replacement surgery. Rehabilitation for patients with hip replacement, we conducted a study on the topic "the effectiveness of educational intervention on rehabilitation knowledge and practice among patients after hip replacement surgery at Thanh Hoa General Hospital in 2021" with the objectives: To examine the effectiveness of educational intervention on rehabilitation knowledge and practice among patients after hip replacement surgery at Thanh Hoa General Hospital.

\section{PARTICIPANTS AND METHODS}

\subsection{Research setting and duration}

Research setting: Department of Trauma Surgery, Thanh Hoa General Hospital.

Research period: from January 2021 to May 2021

2.2. The participants of the study: Patients after hip replacement surgery.

\subsubsection{Inclusion criteria}

- Have completed medical records,

- Be followed up after surgery.

- Patient can communicate in Vietnamese

\subsubsection{Exclusion criteria}

- The patient had previous hip replacement surgery on the other side.

- The patient did not fully participate in the intervention.

\subsection{Research design}

Using the one-group educational intervention method with comparison baseline and post intervention data.

\subsection{Sample size and sampling method}

\section{Sample size}

During the period from January to May 2021, 55 patients who met the inclusion criteria for participating in the study were selected.

\section{Sampling}

Convenience sampling technique was used to collect the patients with hip 
replacement surgery. They were being treated at the Department of Trauma Surgery, Thanh Hoa General Hospital during the data collection period from January 2021 to May 2021.

\subsection{Data collection procedure}

Data collection was performed at two time points using the same measurements. The knowledge and practice of the study population were measured at baseline post intervention health education (T1 - day 1 after surgery) and the knowledge and practice of the after the health education intervention (T2 - before being discharged from the hospital). The selected eligible patients were explained about the purpose of the study and invited to participate in the study. The patient agrees to participate in the study, he/she signed the consent form and participate completely the intervention program.

\subsection{Educational intervention}

The participants were the patient after the 1st day of hip replacement surgery. They were being treated at Department of Trauma Surgery, Thanh Hoa General Hospital.

The researcher was facilitators to perform education intervention program for patients in all sessions ensuring consistency in the contents and methods of educational intervention on mobility rehabilitation after hip replacement surgery. The collaborators supported and performed data collection.

Duration of the intervention program: Daily in the morning from 7:00 a.m. to 9:30 a.m. was the time for usual care and treatment activities. The patients were provided the bedside examination, infusions, bandage change. The rest of the time was spent on instructing the patients in exercise and rehabilitation after surgery. Therefore, the research process was mainly conducted from 9:30 a.m. to 10:30 a.m. in the mornings from Monday to Friday. The duration of a educational intervention sessions lasted 30 to 40 minutes each day.

The content of educational intervention was based on the guidance document of the Ministry of Health No. 3109/QDBYT dated 19/08/2014 and the contents of the hip rehabilitation program after hip replacement surgery by two authors $\mathrm{Ha}$ Hoang Kiem and Luong Anh Tho Research at hospital 175 and Institute of Trauma and Orthopedics in Ho Chi Minh City.

Interventional program was conducted through handouts, presentation. The interventionist explained to provide knowledge for the patient to understand when to start rehabilitation, purpose of rehabilitation, the procedures with explanations and illustrations

\subsection{Measurements}

The questionnaire and checklists were developed based on the guidance of the Ministry of Health No. 3109/QD-BYT [5]. The content of hip rehabilitation program after hip replacement surgery was authored by two authors, Ha Hoang Kiem and Luong Anh Tho studied at the 175 hospital and the Ho Chi Minh City Institute of Trauma and Orthopedics [6].

The questionnaire includes:

Part A: Demographic characteristics of the study participants with questions from A1 to A6.

Part B: Clinical characteristics of the participants with questions from B1 to B4

Part C: Questions of participants' knowledge of rehabilitation after hip 
replacement surgery with questions from C1 to C8

Part D: Checklist to evaluate the participants' practice of rehabilitation after hip replacement surgery with questions from D1 to D5.

The measurement was used uniformly for time points of pre-intervention (T1) and post-intervention (T2).

Evaluation criteria: Calculating the total knowledge score and take the cut-off point of $50 \%$ to classify the knowledge of the participants. The total scores of knowledge were 19, in which, the participants with a total score higher than 9 points were classified in the group with sufficient knowledge, and conversely, those with a knowledge score less than 9 points were grouped with insufficient knowledge. The total score of practice were 19. Calculating the practice total score and take the 50\% cut-off to classify the practice. Research participants with a total score of practice higher than 9 were classified into the group of sufficient practice, and conversely, those with a knowledge score of less than 9 points were grouped with insufficient practice.

\subsection{Data analysis}

The data were collected and processed using SPSS 20.0 software. General information about the participants was analyzed by descriptive statistics including frequency, percentage.

The knowledge and practice improvement after the intervention compared to the baseline was based on comparing the difference in mean scores (X $\left.{ }^{-} \pm S D\right)$ of knowledge and practice. Percentage of participants according to the classification of knowledge and practice levels, percentage of patients with sufficient knowledge and practice by each content were conducted. The t-test was used to compare two mean values.

\subsection{Ethical consideration}

The study was approved by the Scientific Council and the Institutional Review Board of Nam Dinh University of Nursing, Decision No. 12/QD-DDN. The approval was conducted from Thanh Hoa Provincial General Hospital. All research participants were explained specifically about the purpose and content of the research and voluntarily agree to participate and cooperate in the research process. The participants had the right to refuse to participate in the study or to terminate the study at any stage of the study. All participant information was kept confidential and used for research purposes only.

\section{RESULTS}

\subsection{Participant characteristics}

The mean age of the study particpants was $55.31 \pm 10.31$ with the oldest being 73 and the lowest being 23 . In which, the highest proportion with $81.8 \%$ were patients in the age group from 40 to 69 . Patients in the age group higher than 70 accounted for the lowest rate of $7.3 \%$. Most of them were male $(80.0 \%)$, living in rural areas $(85.5 \%)$.

Patients with occupations as farmers accounted for the highest rate of $56.4 \%$, the group of patients working as workers accounted for $7.3 \%$, the group of retire accounted for $14.5 \%$, the group of cadres and civil servants accounted for $12.7 \%$, slightly higher than other occupation groups of $9.1 \%$.

Patients with lower secondary education accounted for $43.6 \%$, the lowest in the group with intermediate, college and 
university degrees accounted for $16.4 \%$, the group of patients with primary and high school accounted for $20 \%$.

The main cause of hip replacement was aseptic necrosis of the femoral head with $49.1 \%$ and fracture of the femoral neck with $36.4 \%$.

Comorbidities included cardiovascular diseases with 4 patients $(7.3 \%)$, hypertension with 7 patients $(12.7 \%)$, diabetes with 6 patients $(10.9 \%)$. Total hip replacement was the most applied in 48 patients, accounting for $87.3 \%$, the remaining $12.7 \%$ was partial hip replacement. $100 \%$ joints were without cement

3.2. The knowledge and practice improvement in rehabilitation of patients undergoing hip replacement surgery after the educational intervention $(n=55)$

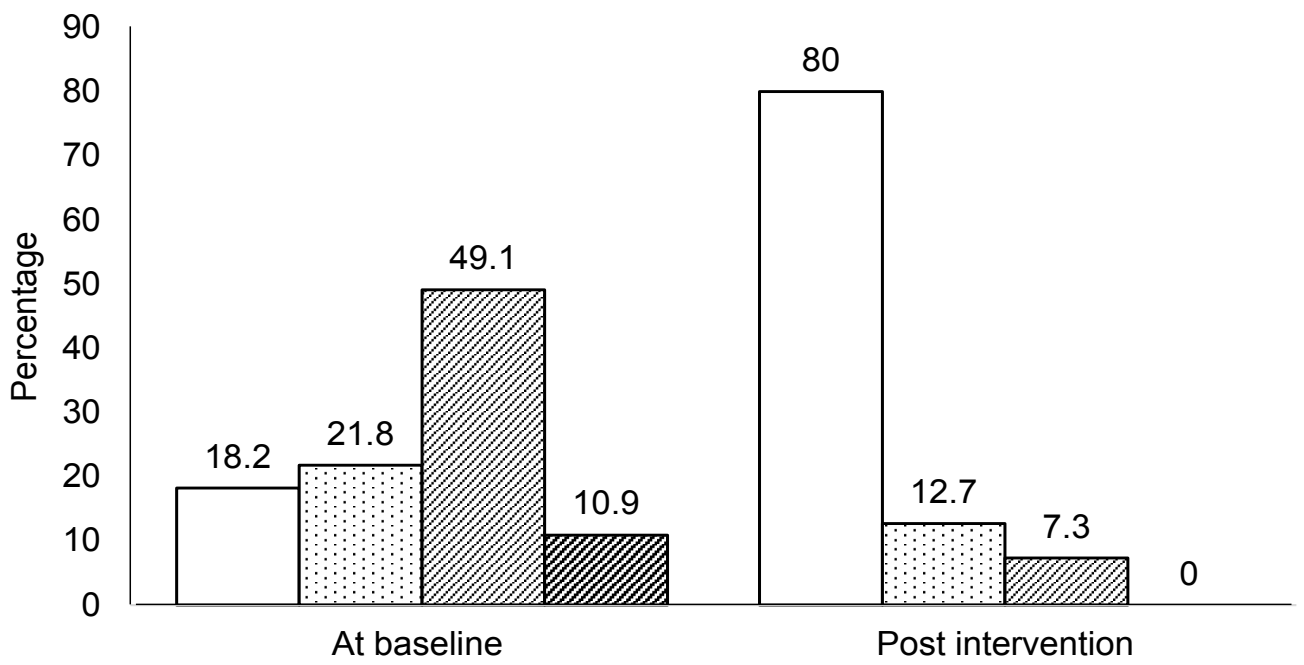

口Day 1 after surgery $₫$ Day 2 after surgery $ఐ$ Day 3 after surgery $₫$ Not sure

Figure 1. The knowledge improvement on the time of rehabilitation after surgery

The figure 1 reseuls indicated that the majority of patients answered the time of rehabilitation was from the 3rd day after surgery, accounting for $49.1 \%$, only $18.2 \%$ of patients answered at the right time for rehabilitation at day 1 postoperation. The rate of correct answers increased to $80 \%$ after the intervention.

Table 1. The practice improvement on movements of rehabilitation $(n=55)$

\begin{tabular}{|lcccc|}
\hline & \multicolumn{2}{c}{ Done correct and sufficient movements } \\
& \multicolumn{2}{c}{ Baseline } & \multicolumn{2}{c|}{ Post intervention } \\
& $\mathbf{n}$ & $\%$ & $\mathbf{n}$ & $\%$ \\
\hline Ankle flexion and extension & 16 & 29,1 & 45 & 81,8 \\
\hline Quad squeeze & 8 & 14,5 & 30 & 54,4 \\
\hline Knee flexion and extension & 4 & 7,3 & 21 & 38,2 \\
\hline
\end{tabular}




\begin{tabular}{|lcccc|}
\hline & & \multicolumn{2}{c|}{ Done correct and sufficient movements } \\
\cline { 2 - 5 } & \multicolumn{2}{c}{ Baseline } & \multicolumn{2}{c|}{ Post intervention } \\
& $\mathbf{n}$ & $\%$ & $\mathbf{n}$ & $\%$ \\
\hline Hip packed open and close & 5 & 9,1 & 33 & 60,0 \\
\hline Leg lift & 2 & 3,6 & 27 & 49,1 \\
\hline
\end{tabular}

The results showed that after the intervention the percentage of patients who performed correctly and sufficiently for each movement increased. Of which, it was noteworthy that $81.8 \%$ of the patients performed correct and sufficient flexion and extension of the ankle joint, however, only $38.2 \%$ of the patients performed the knee flexion and extension and $49.1 \%$ of patients performed leg lifts.

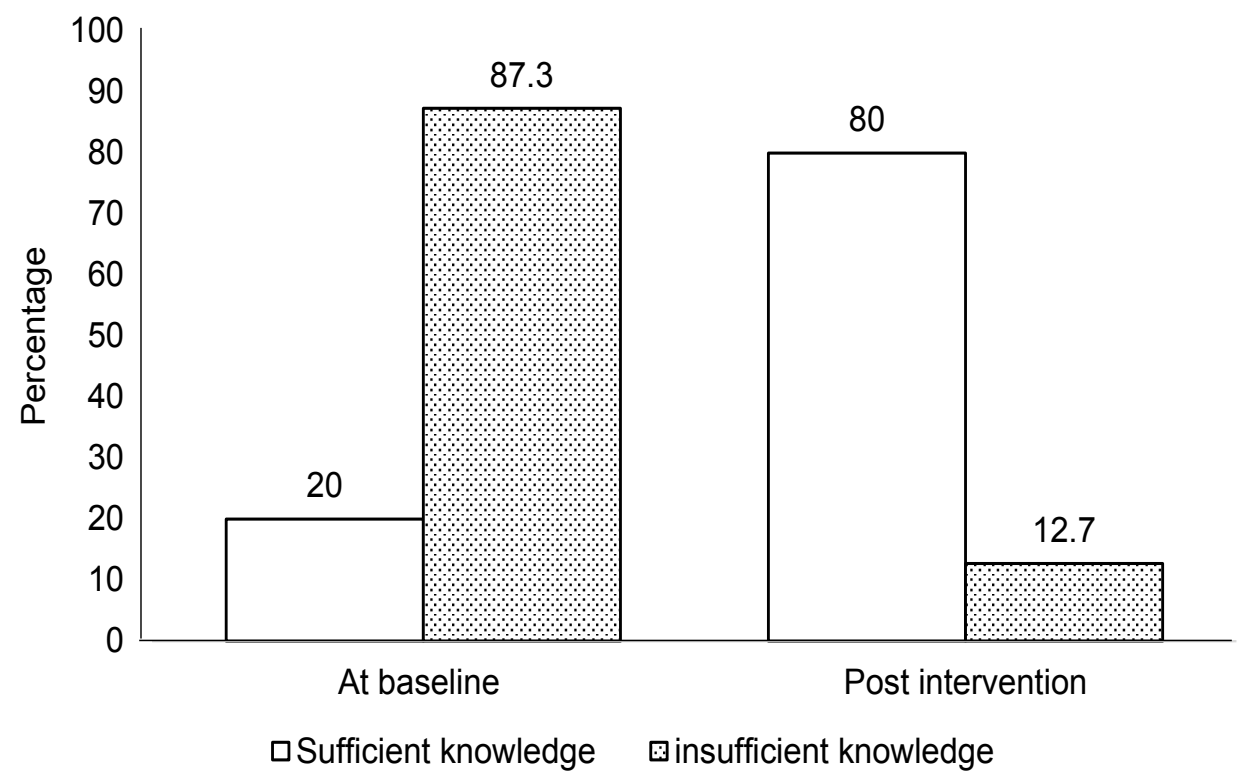

Figure 2. The improvement of knowledge classification on rehabilitation $(n=55)$

Before intervention, the rate of patients with sufficient knowledge reached $20.0 \%$. After the intervention, the percentage of patients with sufficient knowledge increased to $87.3 \%$.

Table 2. The total score of knowledge improvement on rehabilitation $(n=55)$

\begin{tabular}{|cccc|c|}
\hline Score & (Min) & (Max) & Mean (士SD) & \multirow{2}{*}{ p (t-test) } \\
\hline Baseline (T1) & 4 & 13 & $7,96 \pm 1,97$ & \\
\cline { 1 - 3 } Post intervention (T2) & 8 & 18 & $13,18 \pm 2,74$ & $\mathrm{p}=0,00$ \\
\hline
\end{tabular}

Before the intervention, the mean score of the participants' knowledge was 7.96 \pm 1.97 . After the intervention, the average knowledge score of the study participants increased to $13.18 \pm 2.74$, in which, the lowest was 8 and the highest was 18 . The difference was statistically significant with $p<0.01$. 


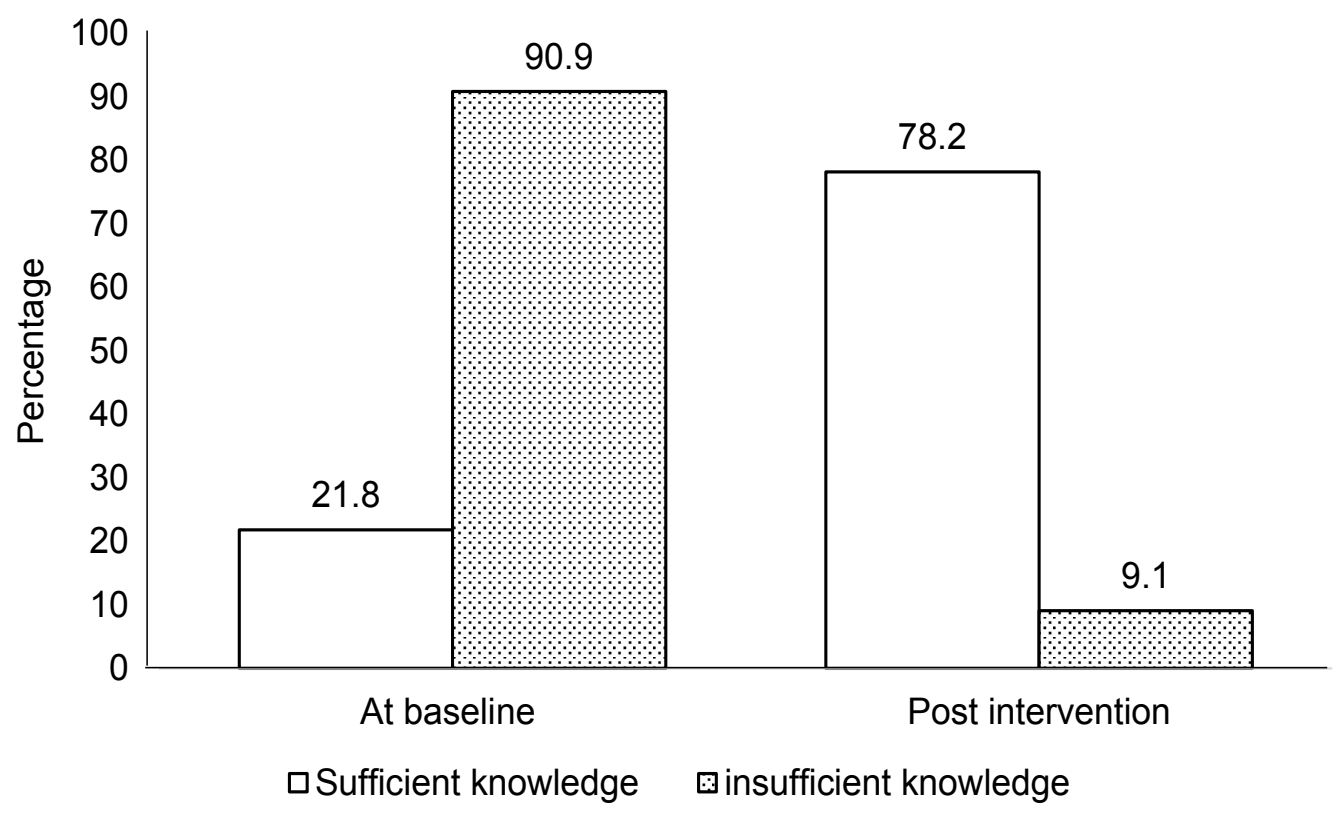

Figure 3. The improvement of practice classification on rehabilitation $(n=55)$

$21.8 \%$ of the patients passed the practice at baseline and increased to $90.9 \%$ after the educational intervention.

Table 3. The total score of practice improvement on rehabilitation $(n=55)$

\begin{tabular}{|ccccc|}
\hline Score & $($ Min) & (Max) & Mean $( \pm \mathbf{S D})$ & $p$ (t-test) \\
\hline Baseline (T1) & 3 & 11 & $6,22 \pm 2,59$ & \\
\hline Post intervention (T2) & 7 & 17 & $13,36 \pm 2,77$ & $\mathrm{p}=0,00$ \\
\hline
\end{tabular}

Before the intervention, the average score of rehabilitation was $6.22 \pm 2.59$, increased to $13.36 \pm 2.77$ after the intervention, in which the lowest score was 7 and the highest score was 17 . The difference between post-intervention and baseline was statistically significant with $p<0.01$.

\section{DISCUSSION}

The results showed that the number of patients who started rehabilitation from day 1 after surgery accounted for a relatively low rate of $18.2 \%$ at baseline. This rate was slightly lower than the results of Nguyen Thi Thuy [4] had $24.2 \%$ and Topçu SY $30.6 \%$ [7] of patients starting to rehabilitation after surgery on the first day. In addition, the research results showed that the majority of patients started rehabilitation from the 3 rd day after surgery, accounting for $49.1 \%$. The reason for the differences because the patient's rehabilitation was dependent on the patient's own efforts. Many patients were afraid of pain and fatigue, so they failed to conduct rehabilitation. In addition, 
patients had the most pain in the first 3 days after surgery [4], but after intervention, the proportion of patients choosing day 1 after surgery as the time to start rehabilitation increased to $80 \%$.

The hip is a large joint with a wide range of movement that is supported by a system of ligaments, joint capsule and hip musculoskeletal system, which is a strong buttock and thigh muscle. Before surgery due to pain, leg sedentary for many months and years, causing muscle atrophy in the buttocks and thighs, making muscles weaker than usual. In order for the hip joint function to be best restored after surgery, the patient needs to start rehabilitation early and properly. Exercises of ankle, knee flexing, extension, quad squeeze, hip open, close were applied by researchers and brought about positive results such as the study of authors Ha Hoang Kiem and Luong Anh Tho [6], Huynh Thi Truc Lam, Le Huy Hoa, Ann Henderson [8], Nguyen Thanh Dieu et al [9]. In the study, before the intervention, the percentage of patients who performed correctly and sufficiently all movements was quite low: ankle flexing, extension with $29.1 \%$; quadriceps muscle contraction with $14.5 \%$; knee flexion and extension with $7.3 \%$; hip open, close with $9.1 \%$ and leg lift with $3.6 \%$. However, after intervention, this rate increased to: $81.8 \%, 54.4 \%, 38.2 \%, 60 \%$ and $49.1 \%$, respectively. This was the reality of the patient's rehabilitation process and thereby the healthcare providers should pay more attention to the patient's condition, explain to the patient the importance of rehabilitation after surgery. Moreover, the nurses who work closely to the patients should supervise the patient's rehabilitation process to assist the patient and the family during rehabilitation to increase the effectiveness of the intervention. Consequently, these actions may limit complications caused by rehabilitation, and speeding up the recovery process for the patient. After the intervention, the percentage of patients who practiced correctly and sufficiently in each movement increased compared to before the intervention.

Classification of rehabilitation knowledge: before the intervention, 20\% of the study participants had sufficient knowledge and $80 \%$ of the study participants insufficient knowledge. Measurement on the patients' knowledge about rehabilitation after hip replacement surgery, the redearcher conducted a health education communication intervention by distributing leaflets and consulting directly with patients in the ward. After the intervention, there were $87.3 \%$ of the study participants with sufficient knowledge. This rate showed that the intervention improved knowledge for the study participants. The percentage of patient with sufficient knowledge increased from $20 \%$ before the intervention to $87.3 \%$ after the intervention. At the time before the intervention, the average knowledge score of the participants was $7.96 \pm 1.97$, after the intervention the mean score of knowledge increased to $13.18 \pm 2.74$ in which, the lowest score was 8 and the person with the highest score was 18 . The difference is statistically significant with $p<0.01$.

Classification of rehabilitation practice: before the intervention, only $21.8 \%$ of the participants achieved right and correct practice and $78.2 \%$ of participants failed. After being directly instructed in practical exercises on patients, that ratio changed markedly. At the time after intervention, the practice rate was $90.9 \%$. Before the intervention, the average practice score 
of the particpants was $6.22 \pm 2.59$, after the intervention the average practice score increased to $13.60 \pm 2.37$, of which the lowest score reached 9. points and the person with the highest score was 17 . This difference is statistically significant with $p<0.01$.

\section{CONCLUSSION}

From the results of measuring the knowledge and practice on rehabilitation of patients after hip replacement surgery at Thanh Hoa Provincial General Hospital, we draw the following conclusions:

Health education interventions markedly improved the rehabilitation knowledge and practice of patients after hip replacement surgery, specifically:

The mean score of the participants' knowledge at the time after the intervention increased to $13.18 \pm 2.74$ compared to 7.96 \pm 1.97 at baseline $(p<0.01)$. The percentage of participants with sufficient knowledge increased to $80.0 \%$ after the intervention.

The mean score of practice of the participants at the time of intervention increased significantly, reaching $13.60 \pm 2.37$ compared to $6.22 \pm 2.37$ at baseline $(p<0.01)$. The rate of participants with correct and right practice increased with $90.9 \%$ after the intervention.

\section{REFERENCES}

1. OECD (2019), "Hip and knee replacement", in Health at a Glance 2019: OECD Indicators, OECD Publishing,Paris, https://doi.org/10.1787/4dd50c09-en

2. Lavernia C.J, Hernandez V.H and Rossi M.D (2007). Payment analysis of total hip replacement. Current Opinion in
Orthopedics, 2007. 18(1), 23-27. https:// doi.org/10.1097/BCO.0b013e328011a270

3. Tran Thi Van Anh and Nguyen Thanh Huong. Evaluation of changes in quality of life of patients after hip replacement surgery at Viet Duc Hospital 2014. Vietnam Journal of Orthopedic Trauma 2014. Special Issue 2014, 359-367.

4. Nguyen Thi Thuy. The status of rehabilitation of patients after surgery of lower extremities fracture at Nam Dinh Province General Hospital. Master Thesis of Nam Dinh University of Nursing.

5. Ministry of Health (2014). Decision No. 3109/QD-BYT dated August 19, 2014 on the promulgation of professional documents "Guidelines for diagnosis and specialized treatment of rehabilitation". Hanoi, 148.

6. Ha Hoang Kiem and Luong Anh Tho. The effectiveness of hip rehabilitation after hip replacement surgery. Journal of Military Medicine (6), 129- 134.

7. Topçu S.Y (2009), "Evaluating of Postoperative Mobilization of Surgical Patients", Türk Cerrahive Ameliyathane Hemşireliği Kongresi Kitabı. 3, pp. 89-91. doi: 10.3233/BMR-2010-0266.

8. Huynh Thi Truc Lam, Le Huy Hoa and Ann Henderson (2019). Pain relief and rehabilitation for patients after hip replacement surgery. Medical Journal (University of Medicine and Pharmacy at Ho Chi Minh City) 23(5), 165- 169.

9. Nguyen Thanh Dieu et al (2014). The nursing care and rehabilitation after partial hip replacement surgery in the elderly at the Military Central Hospital 108. Vietnam Journal of Orthopedic Trauma, special issue $2013,320-326$. 